\title{
Effect of Gender on Atrial Electrophysiologic Changes Induced by Rapid Atrial Pacing and Elevation of Atrial Pressure
}

\author{
HUNG-FAT TSE, M.D., HAKAN ORAL, M.D., FRANK PELOSI, M.D., \\ BRADLEY P. KNIGHT, M.D., S. ADAM STRICKBERGER, M.D., \\ and FRED MORADY, M.D.
}

From the Department of Internal Medicine, Division of Cardiology, University of Michigan Medical Center, Ann Arbor, Michigan

\begin{abstract}
Atrial Electrical Remodeling. Introduction: The incidence of atrial fibrillation is greater in men than in women, but the reasons for this gender difference are unclear. The purpose of this study was to evaluate the effects of gender on the atrial electrophysiologic effects of rapid atrial pacing and an increase in atrial pressure.

Methods and Results: Right atrial pressure and effective refractory period (ERP) were measured during sinus rhythm and during atrial and simultaneous AV pacing at a cycle length of 300 msec in 10 premenopausal women, 11 postmenopausal women, and 24 men. The postmenopausal women were significantly older than the premenopausal women $(61 \pm 8$ years vs $34 \pm 10$ years; $P<0.01)$. During sinus rhythm, mean atrial ERP in premenopausal women was shorter $(211 \pm 19 \mathrm{msec})$ than in postmenopausal women and age-matched men $(242 \pm 18$ msec and $246 \pm 34$ msec, respectively; $P<0.05)$. Atrial ERPs in all patients shortened significantly during atrial and simultaneous AV pacing. However, the degree of shortening during atrial pacing $(43 \pm 8 \mathrm{msec}$ vs $70 \pm 20 \mathrm{msec}$ and $74 \pm 21 \mathrm{msec} ; P<0.05)$ and during simultaneous AV pacing $(48 \pm 16$ msec vs $91 \pm 27$ msec and $84 \pm 26$ msec; $P<0.05)$ was significantly less in premenopausal women than in postmenopausal women or age-matched men.

Conclusion: The results of this study demonstrate a significant gender difference in atrial electrophysiologic changes in response to rapid atrial pacing and an increase in atrial pressure. The effect of menopause on the observed changes suggests that the gender differences may be mediated by the effects of estrogen on atrial electrophysiologic properties. (J Cardiovasc Electrophysiol, Vol. 12, pp. 986-989, September 2001)
\end{abstract}

gender, atrial fibrillation, atrial refractory period

\section{Introduction}

In the Framingham Study, after adjustment for age and other risk factors, atrial fibrillation (AF) occurred $50 \%$ more often in men than in women. ${ }^{1}$ The reasons for this gender difference are unclear. An experimental study demonstrated that $17 \beta$-estradiol attenuates the shortening of atrial refractoriness that occurs after rapid atrial pacing. ${ }^{2}$ A recent clinical study demonstrated significant gender and age differences in the behavior of induced $\mathrm{AF}$ and in the shortening of atrial refractoriness after an episode of AF. ${ }^{3}$ These studies suggested that estrogen may attenuate atrial electrical remodeling, thereby decreasing the susceptibility to AF. However, the relationship between gender and atrial electrophysiologic properties in humans is unclear. Therefore, the goal of the present study was to evaluate the effects of gender and menopause on the atrial effects of rapid pacing and an increase in atrial pressure.

\section{Methods}

\section{Subject Characteristics}

The present study is a retrospective analysis of the data obtained in a prior study of atrial mechanoelectrical feed-

Address for correspondence: Fred Morady, M.D., Department of Internal Medicine, Division of Cardiology, University of Michigan Medical Center, 1500 East Medical Center Drive, B1F245, Ann Arbor, MI 49109-0022. Fax: 734-936-7026; E-mail: fmorady@umich.edu

Manuscript received 26 March 2001; Accepted for publication 3 July 2001 back. ${ }^{4}$ Women who were undergoing hormone replacement therapy or oral contraceptive therapy or who had undergone oophorectomy were excluded from the present analysis. In addition, patients in the prior study in whom the effects of verapamil were tested were excluded. With these exclusion criteria, 45 of the 62 subjects in the prior study were selected for inclusion in the present study.

The study population consisted of 45 patients who underwent radiofrequency catheter ablation of paroxysmal supraventricular tachycardia. None of the study subjects had structural heart disease or a known history of AF. There were 24 men and 21 women. Among the women, 10 were premenopausal and 11 were postmenopausal. The criterion for menopause was amenorrhea for $>12$ months, not attributable to pregnancy. The mean age of the postmenopausal women $(61 \pm 8$ years; mean $\pm \mathrm{SD})$ was significantly greater than the mean age of the premenopausal women ( $34 \pm 10$ years; $\mathrm{P}<0.01$ ). The male patients were divided into two subgroups, according to age, such that the mean ages were matched to the mean ages of the premenopausal and postmenopausal women. Their clinical characteristics are summarized in Table 1 . The women were significantly shorter than the men. However, there were no significant differences in body weight, body surface area, left ventricular ejection fraction, or left atrial size among the four groups of subjects.

\section{Electrophysiologic Procedures}

The electrophysiologic procedures were performed with the patient in the fasting state after informed consent was 
TABLE 1

Clinical Characteristics

\begin{tabular}{|c|c|c|c|c|}
\hline & Premenopausal Women & Young Men & Postmenopausal Women & Elderly Men \\
\hline No. & 10 & 12 & 11 & 12 \\
\hline Age (years) & $34 \pm 10$ & $36 \pm 16$ & $61 \pm 8$ & $60 \pm 9$ \\
\hline Height $(\mathrm{cm})$ & $163 \pm 14 *$ & $176 \pm 16$ & $160 \pm 25^{*}$ & $177 \pm 13$ \\
\hline Body surface area $\left(\mathrm{m}^{2}\right)$ & $1.9 \pm 0.3$ & $2.0 \pm 0.2$ & $1.8 \pm 0.2$ & $2.0 \pm 0.3$ \\
\hline Left ventricular ejection fraction & $0.61 \pm 0.03$ & $0.63 \pm 0.04$ & $0.62 \pm 0.02$ & $0.62 \pm 0.03$ \\
\hline Left atrial size $(\mathrm{cm})$ & $3.4 \pm 0.4$ & $3.6 \pm 0.3$ & $3.5 \pm 0.5$ & $3.7 \pm 0.2$ \\
\hline
\end{tabular}

Variables are expressed as mean $\pm 1 \mathrm{SD}$.

$* \mathrm{P}<0.05$ vs age-matched men

obtained. All antiarrhythmic drug therapy was discontinued at least five half-lives before the procedure. Three quadripolar electrode catheters were inserted into a femoral vein and initially positioned in the high right atrium, His-bundle position, and right ventricular apex. Several ECG leads and the intracardiac electrograms were displayed on an oscilloscope and recorded on optical disk. Pacing was performed with a programmable stimulator using stimuli that had a duration of $2 \mathrm{msec}$.

\section{Study Protocol}

The study protocol was approved by the Human Research Committee and was performed upon completion of the radiofrequency catheter ablation procedure, which was successful in all patients. The details of the study protocol were described previously. ${ }^{4}$ In brief, a quadripolar electrode catheter was positioned in the right atrial appendage such that the pacing threshold was $<1.0 \mathrm{~mA}$. Mean atrial pacing threshold was $0.7 \pm 0.2 \mathrm{~mA}$. Pacing was performed at $3 \times$ threshold. Another electrode catheter was positioned at the right ventricular apex for ventricular pacing. The tip of a $65-\mathrm{cm}$ guiding sheath was positioned in the right atrium to measure atrial pressure. Mean right atrial pressure and mean blood pressure were recorded during each measurement of atrial effective refractory period (ERP).

Atrial ERP was measured during sinus rhythm by introducing an atrial extrastimulus after every eighth sinus beat at an initial coupling interval of $150 \mathrm{msec}$ and increasing the coupling interval in increments of $5 \mathrm{msec}$ until there was atrial capture. Atrial ERP was defined as the longest S1-S2 coupling interval that failed to result in atrial capture.

Atrial ERP then was measured during atrial and simultaneous $\mathrm{AV}$ pacing at a cycle length of $300 \mathrm{msec}$, in random order. Simultaneous AV pacing (AV interval $=0 \mathrm{msec})$ at a cycle length of $300 \mathrm{msec}$ was used to evoke an acute increase in right atrial pressure, ${ }^{4}$ and right atrial pacing at the same cycle length was used to control for the effect of an increase in rate on atrial ERP. After 3 minutes of continuous pacing at a cycle length of $300 \mathrm{msec}$, an atrial extrastimulus was introduced at a coupling interval of 100 msec. After every eighth drive train stimulus, the coupling interval of the extrastimulus was increased by $5 \mathrm{msec}$, with no pause in the drive train, until the extrastimulus resulted in atrial capture twice in succession.

\section{Statistical Analysis}

Continuous variables are expressed as mean $\pm 1 \mathrm{SD}$. Statistical comparisons were performed with Student's $t$ test. Analysis of variance was used for multiple group comparisons, followed by a Bonferroni-corrected $t$-test. $\mathrm{P}<$ 0.05 was considered statistically significant.

\section{Results}

\section{Hemodynamic Changes}

During sinus rhythm, mean right atrial pressure in premenopausal women, postmenopausal women, and agematched men did not differ significantly (Table 2). Mean right atrial pressure during atrial pacing at a cycle length of $300 \mathrm{msec}$ also did not differ significantly among the four groups of patients (Table 2).

During simultaneous AV pacing at a cycle length of 300 msec, mean right atrial pressure increased significantly compared with during sinus rhythm and atrial pacing, and it increased to a similar degree in the premenopausal women, postmenopausal women, and age-matched men (Table 2).

\section{TABLE 2}

Hemodynamic Changes

\begin{tabular}{|c|c|c|c|c|c|}
\hline & $\begin{array}{l}\text { Premenopausal Women } \\
(\mathbf{n}=\mathbf{1 0})\end{array}$ & $\begin{array}{l}\text { Young Men } \\
(\mathbf{n}=12)\end{array}$ & $\begin{array}{l}\text { Postmenopausal Women } \\
(\mathbf{n}=\mathbf{1 1})\end{array}$ & $\begin{array}{l}\text { Elderly Men } \\
(\mathbf{n}=12)\end{array}$ & P Value \\
\hline \multicolumn{6}{|c|}{ Mean right atrial pressure $(\mathrm{mmHg})$} \\
\hline Sinus rhythm & $2.1 \pm 2.6^{*}$ & $3.2 \pm 2.8^{*}$ & $3.2 \pm 3.0^{*}$ & $2.8 \pm 2.9^{*}$ & $>0.05$ \\
\hline AV pacing & $9.6 \pm 3.2$ & $11.3 \pm 3.6$ & $10.2 \pm 3.5$ & $11.3 \pm 2.7$ & $>0.05$ \\
\hline \multicolumn{6}{|c|}{ Mean changes in right atrial pressure $(\mathrm{mmHg}) \dagger$} \\
\hline Atrial pacing & $2.0 \pm 2.5^{*}$ & $2.1 \pm 3.0^{*}$ & $1.1 \pm 1.9^{*}$ & $1.3 \pm 2.1^{*}$ & $>0.05$ \\
\hline AV pacing & $6.1 \pm 2.5$ & $6.6 \pm 2.2$ & $8.1 \pm 2.3$ & $8.5 \pm 1.9$ & $>0.05$ \\
\hline
\end{tabular}

Variables are expressed as mean $\pm 1 \mathrm{SD}$.

$* \mathrm{P}<0.05$ vs pacing.

$\dagger$ Changes in pressure compared with sinus rhythm. 
TABLE 3

Atrial Effective Refractory Periods

\begin{tabular}{|c|c|c|c|c|c|}
\hline & $\begin{array}{l}\text { Premenopausal Women } \\
\qquad(\mathbf{n}=\mathbf{1 0})\end{array}$ & $\begin{array}{l}\text { Young Men } \\
(\mathbf{n}=12)\end{array}$ & $\begin{array}{l}\text { Postmenopausal Women } \\
(\mathbf{n}=\mathbf{1 1})\end{array}$ & $\begin{array}{l}\text { Elderly Men } \\
\quad(\mathbf{n}=12)\end{array}$ & P Value \\
\hline Sinus cycle length (msec) & $859 \pm 120$ & $790 \pm 150$ & $890 \pm 113$ & $802 \pm 124$ & $>0.05$ \\
\hline \multicolumn{6}{|l|}{ Mean ERP (msec) } \\
\hline Atrial pacing & $169 \pm 18 *$ & $171 \pm 24^{*}$ & $172 \pm 19^{*}$ & $173 \pm 19^{*}$ & $>0.05$ \\
\hline AV pacing & $163 \pm 20$ & $162 \pm 26$ & $151 \pm 25$ & $149 \pm 28$ & $>0.05$ \\
\hline \multicolumn{6}{|l|}{ Mean changes in ERP msec $\$$} \\
\hline
\end{tabular}

Variables are expressed as mean $\pm 1 \mathrm{SD}$. ERP $=$ effective refractory period.

${ }^{*} \mathrm{P}<0.05$ vs AV pacing; $\dagger \mathrm{P}<0.05$ vs atrial pacing.

$\dagger$ Changes in pressure compared with sinus rhythm.

\section{Atrial Refractoriness}

There were no significant differences in mean sinus cycle length among premenopausal women, postmenopausal women, and age-matched men (Table 3). During sinus rhythm, atrial ERP in premenopausal women was significantly shorter than in postmenopausal women and than in both groups of men (Table 3 ).

Compared with during sinus rhythm, mean atrial ERP shortened significantly during atrial pacing $(\mathrm{P}<0.01)$, with a further shortening during simultaneous $\mathrm{AV}$ pacing in postmenopausal women and men $(\mathrm{P}<0.01$; Table 3$)$. In premenopausal women, mean atrial ERP decreased significantly during atrial pacing, with no further significant change during simultaneous AV pacing (Table 3).

\section{Menopausal Status}

The degree of shortening of atrial ERP during atrial and simultaneous AV pacing was significantly smaller in premenopausal women than in postmenopausal women or in age-matched men (Table 3).

\section{Effect of Age and Height}

Changes in atrial ERP in response to atrial and simultaneous AV pacing were similar in magnitude between the younger and older men (Table 3). Despite the significant difference in height between the postmenopausal women and the age-matched men, there were no significant differences in the changes in atrial ERP in response to atrial or simultaneous AV pacing (Table 3).

\section{Discussion}

\section{Main Findings}

The results of this study indicate that atrial ERP and the response of the atrial ERP to tachycardia and an increase in atrial pressure in women are influenced by menopausal status. In premenopausal women, baseline atrial refractoriness is shorter than in postmenopausal women. Furthermore, the degree of shortening in ERP that occurs during rapid atrial pacing and the elevation of atrial pressure is smaller in magnitude in premenopausal women than in postmenopausal women. If the differences between premenopausal and postmenopausal women were attributable simply to the difference in age between the two groups of women, age-dependent differences in atrial ERP also would be expected in men. However, there were no age-dependent differences in atrial ERP among the men, and changes in atrial ERP in response to tachycardia and elevated atrial pressure in the men did not differ significantly from the postmenopausal women.

These findings suggest that the differences in atrial electrophysiologic characteristics between the premenopausal and postmenopausal women may be attributable to hormones such as estrogen. It is possible that the attenuated shortening of atrial ERP in response to tachycardia and an elevated atrial pressure observed in premenopausal women contributes to the gender difference in the incidence of AF.

\section{Prior Studies}

Prior experimental studies demonstrated that $17 \beta$-estradiol shortens the action potential duration in atrial myocytes without significantly affecting the resting membrane potential. ${ }^{5,6}$ In the present study, the shorter baseline atrial ERP in premenopausal women than in postmenopausal women or in age-matched men is consistent with this finding. However, another experimental study reported that atrial refractoriness prolonged after $17 \beta$-estradiol administration. ${ }^{2}$ The reason for this discrepancy is unclear but may be related to the use of a supraphysiologic dosage of $17 \beta$-estradiol in the latter study.

Pretreatment with $17 \beta$-estradiol in dogs was found to prevent the shortening of atrial refractoriness that otherwise occurs after rapid atrial pacing. A recent clinical study found that the shortening in atrial ERP that occurs after an episode of AF is lesser in magnitude in women than in men. ${ }^{3}$ The results of the present study are consistent with these findings.

\section{Possible Mechanisms}

Experimental studies demonstrated that $17 \beta$-estradiol suppresses the expression of L-type calcium channels and inhibits the L-type calcium current. ${ }^{6-8}$ The resultant reduction in the plateau phase of the atrial action potential might explain why the baseline atrial ERP in the present study was found to be shorter in premenopausal women than in postmenopausal women or in men.

Prior studies in humans $s^{9,10}$ and animals ${ }^{1,12}$ demonstrated that tachycardia-induced shortening of atrial refractoriness is related to intracellular calcium overload and reduction in calcium current. Furthermore, recent clinical ${ }^{4}$ and experi- 
mental ${ }^{13}$ studies showed that calcium channel blockade with verapamil attenuates the shortening of atrial refractoriness that occurs in response to an increase in atrial pressure. It is possible that the calcium channel-blocking properties that estrogen may have in common with verapamil explain the attenuated effects of tachycardia and elevated atrial pressure on atrial refractoriness in the premenopausal women in the present study.

Estrogen also was demonstrated to block the delayed outward potassium current $\left(\mathrm{I}_{\mathrm{Kr}}\right.$ and $\left.\mathrm{I}_{\mathrm{Ks}}\right),{ }^{5,6}$ providing another possible mechanism for the blunted response of atrial ERP due to tachycardia and elevated atrial pressure in premenopausal women.

\section{Study Limitations}

One limitation of this study is that the estrogen plasma concentrations in the premenopausal women may have varied significantly, depending on the timing of the electrophysiologic procedure relative to the menstrual cycle. Furthermore, the retrospective nature of the analysis did not allow measurement of plasma estrogen concentrations.

Another limitation is that all the patients in this study had a history of paroxysmal supraventricular tachycardia and had undergone radiofrequency ablation; therefore, the findings may not apply to normal individuals. Finally, the sample size of this study was small, and the results require confirmation in a larger study.

\section{Conclusion}

There is a significant gender difference in atrial electrophysiologic characteristics that is manifest only in premenopausal women, suggesting that the gender difference may be attributable to estrogen. The effects of estrogen on atrial electrophysiologic characteristics may help to explain the lower incidence of AF among young women. Further studies are needed to clarify the relationship between estrogen and the susceptibility to AF and to determine whether hormone replacement therapy affects the incidence of AF in postmenopausal women.

\section{References}

1. Benjamin EJ, Levy D, Vaziri SM, D’Agostino RB, Belanger AJ, Wolf PA: Independent risk factors for atrial fibrillation in a population-based cohort: The Framingham Heart study. JAMA 1994;271:840-844.

2. Chen YJ, Lee SH, Hsieh MH, Hsiao CJ, Yu WC, Chiou CW, Chen SA: Effects of $17 \beta$-estradiol on tachycardia-induced changes of atrial refractoriness and cisapride-induced ventricular arrhythmia. J Cardiovasc Electrophysiol 1999;10:587-598.

3. Tada H, Sticherling C, Chough SP, Baker RL, Wasmer K, Daoud EG, Pelosi F, Knight BP, Strickberger SA, Morady F: Gender and age differences in induced atrial fibrillation. Am J Cardiol 2001; (In press).

4. Tse HF, Pelosi F, Flemming M, Knight BP, Strickberger SA, Morady F: Effects of simultaneous atrioventricular pacing on atrial refractoriness and atrial fibrillation inducibility: Role of atrial mechanoelectrical feedback. J Cardiovasc Electrophysiol 2001;12:43-50.

5. De Beer EL, Keizer HA: Direct action of estradiol-17 beta on the atrial action potential. Steroids 1982;40:223-231.

6. Nakajima T, Iwasawa K, Oonuma H, Morita T, Goto A, Wang Y, Hazama H: Antiarrhythmic effect and its underlying ionic mechanism of 17 beta-estradiol in cardiac myocytes. Br J Pharmacol 1999;127: 429-440.

7. Tanabe S, Hata T, Hiraoka M: Effects of estrogen on action potential and membrane currents in guinea pig ventricular myocytes. Am J Physiol 1999;277:H826-H833.

8. Meyer R, Linz KW, Surges R, Meinardus S, Vees J, Hoffmann A, Windholz O, Grohe C: Rapid modulation of L-type calcium current by acutely applied oestrogens in isolated cardiac myocytes from human, guinea-pig and rat. Exp Physiol 1998;83:305-321.

9. Daoud EG, Knight BP, Weiss R, Bahu M, Paladino W, Goyal R, Man KC, Strickberger SA, Morady F: Effect of verapamil and procainamide on atrial fibrillation induced electrical remodeling in humans. Circulation 1997;96:1542-1550.

10. Sticherling C, Oral H, Horrocks J, Chough SP, Baker RL, Kim MH, Wasmer K, Pelosi F, Knight BP, Michaud GF, Strickberger SA, Morady F: Effects of digoxin on acute atrial fibrillation-induced changes in atrial refractoriness. Circulation 2000;102:2503-2508

11. Goette A, Honeycutt C, Langberg JJ: Electrical remodeling in atrial fibrillation: Time course and mechanisms. Circulation 1996;94:29682974.

12. Tieleman RG, De Langen CDJ, Van Gelder IC, de Kam PJ, Grandjean J, Bel KJ, Wijffels MC, Allessie MA, Crijns HJ: Verapamil reduces tachycardia-induced electrical remodeling of the atria. Circulation 1997;95:1945-1953.

13. Zarse M, Stellbrink C, Athanatou E, Robert J, Schotten U, Hanrath P: Verapamil prevents stretch-induced shortening of atrial effective refractory period in Langendorff-perfused rabbit heart. J Cardiovasc Electrophysiol 2001;12:85-92. 